\title{
Parenting Stress and Social Support among Cross-National Families
}

\author{
Yajuan Xiang \\ University of Southern Indiana \\ Indiana, USA \\ James Hoot \\ The State University of New York at Buffalo \\ New York, USA \\ Jill Raisor \\ University of Southern Indiana \\ Indiana, USA
}

\begin{abstract}
As a result of globalization, the number of cross-national couples in the United States is on the rise. However, a primary conclusion of the past research on such marriages has been subsumed under general intermarriages. The results of these studies focused on the challenges confronted by couples in potential conflicts caused by their disparate cultural backgrounds. Place of residence, language issues, societal stereotypes, extended families, and cultural complexity were identified in most qualitative studies as stressors most likely to contribute to a greater levels of parenting stress. A relatively small proportion of the literature in this area has focused on childrearing which was recognized as a flashpoint for more conflicts in these marriages. Thus, this study sought to compare the level of parenting stress and social support between cross-national and traditional families. Purposeful sampling was used to recruit 159 parents from both crossnational (82) and traditional (77) families in Western New York area. This study adopted the Parental Stress Scale (PSS), Family Support Scale (FSS), and self-developed questionnaire. Basic statistics and multiple regression analysis were applied. The results suggested parents from crossnational families do not experience higher level parenting stress comparing to their counterparts in traditional families. However, the number of children and cultural influences were found significantly related to parenting stress in cross-national families. In other words, the more children in a family and more distinct cultural difference between couples strongly contribute to the level of parenting stress among cross-national families.
\end{abstract}


Yajuan Xiang et al.

\section{Definitions}

A cross-national couple refers to a heterosexual married pair from two different countries who speak different native languages. The term non-cross-national couple is in contrast to crossnational couple referring to a heterosexual married pair who share the same country of origin and native language.

\section{Introduction}

Growing up in different cultural traditions, intercultural couples are likely to have divergent beliefs and practices regarding parent-child relationships, parental roles, discipline and punishment, and daily routines of children (Coll \& Pachter, 2002; Cools, 2006; Jambunathan, Burts, \& Pierce, 2000; Romano, 2008). When intercultural couples fail to reconcile their childrearing differences, the development of children, parent-child relations, and couple relations are placed at a higher risk of dysfunction (Bhugun, 2017; Bustamante, Nelson, Richard C. Henriksen, \& Monakes, 2011).

Cross-national couples, a subcategory of intercultural marriage, experience greater cultural variance regarding childrearing within the family unit (Baltas \& Steptoe, 2000; Bustamante et al., 2011; Crippen \& Brew, 2007). Cross-national families are typically composed of couples who have distinctive cultural roots and speak different native languages. In most cases, those couples are more likely to be ethnically, culturally, and racially different. They may not share fundamental cultural values and norms and have differing nationalities and citizenships.

Although data regarding such populations is still not available, in 2015, a total of 1,051,030 persons became legal permanent residents of the United States (US). Of these, 265,367 (25.2\%) gained their residence as a spouse of an American citizen (Baugh \& Witsman, 2017). This indicates increasingly diverse family structures in the US (Bikel \& Mandarano, 2012). Despite expected cultural differences in these diverse families, however, little is known about how such differences might impact parental practices. This is primarily because related research to date has included cross-national families in discussion of general intermarriage studies, such as "interracial," "interethnic," and "intercultural" marriages. Those research tended to focus on marital relationships rather than childrearing (Cottrell, 1990; Lee \& Fernandez, 1998; Seto \& Cavallaro, 2007). Of these studies, most were conducted on small samples, using qualitative research methods (Egan, 1995; Wieling, 2003).

Data from such studies suggest that cross-national couples experience unique stressors. These include: gaining legal status for foreign-born spouses, linguistic acquisition, family ties, societal reactions and cultural complexity (Seto \& Cavallaro, 2007), seeking social support, adjusting to the new culture, and reframing cultural differences shared within couples (Baltas \& Steptoe, 2000; Bustamante et al., 2011; Crippen \& Brew, 2007; Kuramoto, Koide, Yoshida, \& Ogawa, 2017). Moreover, childrearing has been shown to be a major stressor among cross-national couples, compared to their same-culture counterparts (Bustamante et al., 2011; Crippen \& Brew, 2007, 2013; Djurdjevic \& Roca Girona, 2016). Thus, there is a need to distinguish cross-national 
couples from general intermarriage population and investigate whether those unique stressors in cross-national families would contribute to the couples' childrearing experiences.

\section{Place of Residence}

\section{Literature Review}

In a cross-national marriage, at least one spouse must learn how to live and function in a foreign country. Living abroad is often associated with culture shock, homesickness, feelings of loneliness, social isolation, frustration, inadequacy, depression, and a feeling of being caught between two cultures (Adams, 2004; Molina, Estrada, \& Burnett, 2004; Sinha, 1998; Wieling, 2003). Foreign-born spouses often feel isolated and receive limited social support (Imamura, 1990). They also tend to have difficulties in obtaining educational resources to teach their heritage culture and language to their children (Kuramoto et al., 2017). In contrast, native-born spouses have less need to change habitual ways and acculturate into the spouses' culture (Rosenblatt, 2009; Wieling, 2003) and have greater power to make family decisions (Romano, 2008).

\section{Language}

Languages used in cross-national families have a significant impact on couples. Foreignborn spouses who have limited language proficiency have greater difficulties with socialization, (Imamura, 1990; Romano, 2008; Turney \& Kao, 2009), career options, adjusting to a new culture, and childrearing (Ali, 2008; Breger \& Hill, 1998; Yaman, Mesman, van IJzendoorn, \& Bakermans-Kranenburg, 2010). In an example from Romano (2008, p. 129), an American wife living in Germany commented that she had "lost [her] personality" and "sense of humor" and that "there is a danger in teasing or making jokes." Further, foreign-born spouses with children reported difficulties communicating with teachers, helping with homework, and getting involved in school activities (Kuramoto et al., 2017).

\section{Societal Attitudes and Extended Families}

Societies often have negative attitudes toward cross-national couples based on a set of intertwined factors including: cultural, race, age, religion, and socioeconomic status differences within couples, historical relationships and distance between the two countries, fluency of shared language, and perceptions of gender and gender roles in one society (Bystydzienski, 2011; Kalmijn, 1998; Kim \& McGoldrick, 1998; Yang \& Lu, 2010). In Japan, for example, children with mixed appearance and heritage were being targeted in school (Kuramoto et al., 2017). When such attitudes and reactions are from extended families and friends, they can be even more severe because of the closeness of relationships (Bystydzienski, 2011; Mcfadden \& Moore, 2001; Rosenblatt, 2009).

\section{Clash of Cultural Values}

Cross-national couples have distinctive cultural backgrounds. Those differences are often viewed as exciting and interesting at the beginning of the marriage but may lead to increased emotional difficulties and marital conflicts (Baltas \& Steptoe, 2000; Bystydzienski, 2011; Cohen, 1982; Cools, 2006). Such conflicts often revolve around: different childrearing beliefs and practices, relationships among family members (Bystydzienski, 2011), parent-child relationships, roles and responsibilities between fathers and mothers (Romano, 2008), children's identity, (Caballero, Puthussery, \& Edwards, 2008), health care, school preference (Kuramoto et al., 2017), parent's interactions with schools, and child discipline methods (Bustamante et al., 2011; Cools, 
Yajuan Xiang et al.

2006). These disagreements and conflicting childrearing beliefs and practices may complicate children's development in the long run.

Every spouse comes into parenthood with a different set of personal and social experiences that require some adjustment and accommodation. Besides those differences, cross-national couples undergo unique stressors due to the nature of their marriages. Place of residence, language, societal attitudes, extended families, cultural complexity, and intercultural parenting provide extra substance for potential stressors. These stressors are likely to complicate their perceptions of parenthood and raising mixed-heritage children. Thus, the purpose of this study is to better understand differences in perceived parenting stress and social support between cross-national and non-cross-national families in the US. In addition, potential effects of demographic characteristics as well as unique cross-national stressors on parenting stress among cross-national families were investigated. The following research questions guided this study.

1. What are the differences in perceived parenting stress and social support between crossnational and non-cross-national families?

2. What is the relationship between variables of interest (parent gender, parent age, SES, yearly income, child age, and number of children) and perceived parenting stress by family types?

3. What is the relationship between cross-national family-specific variables (place of residence/status, language proficiency, societal attitude, cultural complexity, and social support) and perceived parenting stress?

\section{Participants}

\section{Method}

In this study, cross-national couples consisted of heterosexual married pairs. One member of the pair was a US-born citizen with English as native language and the other member was a foreign-born national with a non-English native language, residing in the US. Participants from the non-cross-national families were heterosexual married pairs of two US-born citizens with English as their native language. In total, 83 families from both cross-national (43) and non-crossnational (40) families agreed to participate in this study. A sample of $159\left(n_{\text {cross-national }}=82 ; n_{\text {non- }}\right.$ cross-national $=77)$ participants completed the questionnaires. Of these, $79(49.7 \%)$ were male and 80 $(50.3 \%)$ were female. The average age of the participants was 39.03 years. Ages ranged from 2454. The ethnic/racial make-up of the sample was 33 Asian (20.8\%), 115 White (72.3\%) or Caucasian, 9 Hispanic or Latino (5.7\%), 1 American Indian or Alaska Native (.6\%), and 1 participant of more than one ethnicity/race (.6\%). All participants had a minimum of high school education; 76 possessed graduate/professional training education $(47.8 \%)$; 67 participants held a Bachelor's degree (42.1\%); 7 (4.4\%) and 9 (5.7\%) people had a partial college education and a high school diploma, respectively.

The average number of children ranged from 1-8 with a mean of 1.96 . The average age of children within cross-national families was 4.28 , and 4.93 in non-cross-national families. There was a large gap in family yearly income among all families. Incomes ranged from \$7,500$\$ 175,000$. On average, non-cross-national families had a higher income compared to crossnational families. The average family socioeconomic status (SES) score was 47.54 (score range 866) with 23 as the lowest and 66 as the highest. Between the two family types, there were no significant differences on any demographic variables.

ISSN 2325-6389 
Within cross-national families, there were 41 native-born spouses and 41 foreign-born spouses. Native-born spouses tended to be older than foreign-born spouses, with average ages of 40.28 and 37.93 respectively. Both native and foreign-born spouses possessed higher education achievement where $37(90.2 \%)$ and $35(85.4 \%)$ participants had university/college and above education. Among 41 foreign-born spouses, $8(19.5 \%)$ were males and $33(80.5 \%)$ were females.

The majority of foreign-born spouses were Asian: $28(68.3 \%)$ from 8 countries $^{1}$, followed by $8(19.5 \%)$ spouses from 7 European countries ${ }^{2}$. There were $2(4.9 \%)$ spouses from Mexico and $3(7.3 \%)$ from South American countries ${ }^{3}$. In terms of primary languages, 17 (41.5\%) foreign-born spouses spoke Mandarin and/or Cantonese, 4 (9.8\%) spoke Japanese, 3 (7.3\%) Spanish and 1 (2.4\%) identified as having more than one primary language. Sixteen participants $(39.0 \%)$ spoke 15 other languages ${ }^{4}$. The average English proficiency score was 3.34 (score range 1-4) where 2 (4.9\%) participants reported basic English ability, 4 (9.8\%) competent, 13 (31.7\%) proficient, and $22(53.7 \%)$ fluent.

\section{Recruitment}

Given the relatively small population of cross-national couples, snowball sampling was used for recruitment. The total recruitment process took approximately 2 months. During this time, flyers were distributed to professional and personal contacts at institutions, organizations ${ }^{5}$ and through listservs ${ }^{6}$ primarily in the Western New York area. Potential participants were asked to contact the senior researcher for details. Snowball sampling is sometimes questioned regarding ethnical concerns when participants are asked to provide contact information of peers. To avoid this, participants in this study were only asked to forward the senior researcher's contact information to peers. A screening process was then conducted based on the given criteria.

\section{Demographics}

\section{Measures}

A researcher-designed demographic survey (DS) contained 15 questions including gender, age, ethnicity, country of origin, years living in the US, current status in the US, primary/first/mother language, English ability, yearly income, occupation, education level, number of children in the household, ages of children, and number of children referred to behavioral and developmental interventions in the household. Data gathered through this questionnaire was used to test Research Question 1 regarding potential differences in levels of parenting stress and social support between and within family types.

\section{Parental stress}

The Parental Stress Scale (PSS) (Berry \& Jones, 1995) is a self-report scale which consists of 18 items covering a range of topics including closeness with children, satisfaction with the parental role, and positive aspects of parenting (e.g., self-enrichment, emotional benefits), as well as negative components of parenting (e.g., demands on resource, opportunity costs). Respondents were asked to rate on a 5-point Likert scale ranging from "strongly disagree" to "strongly agree." The satisfactory level of internal reliability and test-retest reliability were shown as .83 and .81 . This scale was tested with a multicultural population and produced a .72 reliability (Baker, Perilla, \& Norris, 2001). 
Yajuan Xiang et al.

\section{Family support}

The Family Support Scale (FSS) (Dunst, Jenkins, \& Trivette, 1984) was developed based on the ecological theory (Bronfenbrenner, 1994; Bronfenbrenner \& Morris, 2007). It covers four areas of resources from which parents could get help: extended family, kinship members, social units, and human service agencies. The FSS consists of 18 self-report items. Ratings are made on a 5-point Likert scale ranging from "not at all helpful" to "extremely helpful." This scale was designed to measure satisfaction with sources of support and degree of perceived helpfulness. The authors reported both validity and acceptable internal consistency with $\alpha=.77$. The internal reliability of FSS in this study was $\alpha=0.84$.

\section{Societal attitudes and cultural influence}

Two questions were designed to measure the influence of societal attitudes and culture on childrearing. Respondents were asked to rate their views on a 5-point Likert scale ranging from "strongly disagree" to "strongly agree."

\section{Data Analysis}

Due to the relatively small sample size, and since the mothers and fathers were nested within the same families, 7 variables were aggregated as family-level variables including: family type, yearly income, SES, child age, number of children, social support, and parenting stress. To answer Research Question One, an independent sample T-test was employed to test the differences in family-level parenting stress and social support. To examine Research Question Two, Multiple Linear Regression analysis was used. Parent age and gender in relation to parenting stress were tested at the individual level, where variables of SES, yearly income, child age, and number of children were examined at family-level. For Research Question Three, variables found to be significant contributors in Question 2 were controlled first. Then cross-national family-specific variables: status (being foreign-born or native-born spouse), years living in the US, English proficiency, societal attitude, cultural influence and social support were tested utilizing Hierarchical Regression.

\section{Comparison between Family Types}

\section{Results}

Parenting stress and social support scores from each family type were aggregated between father and mother in the same household ( $n_{\text {cross-national }}=43$ and $\left.n_{\text {non-cross-national }}=40\right)$. Parents from cross-national families scored from 7 to 31.5 on PSS, where scores in non-cross-national families ranged from 5.5 to 35 . The mean PSS score was $\mathbf{M}_{\text {cross-national }}=18.05, S D_{\text {cross-national }}=5.91$ and $\mathrm{M}_{\text {non-cross-national }}=16.44, S D_{\text {non-cross-national }}=5.93$. Generally, parents from cross-national families scored higher (1.61) on parenting stress than their counterparts. However, the difference between parenting stress score was not statistically significant $t(81)=-1.24, p=.22$.

As for perceived social support, the mean score was $\mathbf{M}_{\text {cross-national }}=2.83, S D_{\text {cross-national }}=.61$ ranged from 1.76 to 4.05 , and $\mathrm{M}_{\text {non-cross-national }}=2.86, S D_{\text {non-cross-national }}=.51$ ranged from 1.85 to 4.36 . The difference in perceived social support between two family types was negligible (.03). No statistically significance in FSS score was found, $t(81)=.17, p=.87$.

\section{Demographic Variables and Parenting Stress by Family Types}

The relationship between demographic parent and family-level variables and parenting stress in both family types was also examined. Parent-level variables (age and gender) were 
addressed first. The DS results indicated that parents in cross-national families were relatively older $\left(M_{\text {cross-national }}=39.09, S D_{\text {cross-national }}=6.42\right)$ than parents in non-cross-national families $\left(M_{n o n-}\right.$ cross-national $\left.=38.09, S D_{\text {non-cross-national }}=6.16\right)$. The correlation between parent age and parenting stress was -.12 $(p=.28)$ and $.077(p=.51)$ in cross-national and non-cross-national families respectively. Older parents experienced less stress in cross-national families but the pattern reversed in non-cross-national families. Although the relationship between parent age and parenting stress showed opposite directions between family types, the relationship was negligible whereas cross-national families were slightly higher than their counterparts.

For the parent gender variable between family types, there were $51.2 \%$ males and $48.8 \%$ females in cross-national families and $48.1 \%$ males and $51.9 \%$ females in non-cross-national families. The mean parenting stress score was $M_{\text {cross-national males }}=17.02\left(S D_{\text {cross-national males }}=7.55\right)$, and $\mathbf{M}_{\text {cross-national females }}=19.68\left(S D_{\text {cross-national females }}=7.81\right.$. In cross-national families, females generally experienced higher parenting stress (2.65) than males. In non-cross-national families, the difference between genders on perceived parenting stress was relatively smaller (.26) among

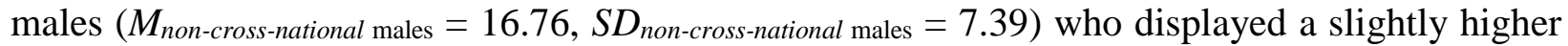
stress level than females $\left(M_{\text {non-cross-national females }}=16.5, S D_{\text {non-cross-national females }}=6.77\right)$. Overall, parents in cross-national families had higher stress scores than those in non-cross-national families regardless of gender. Parent gender was positively related to parenting stress $r=.17(p=.12)$ in cross-national families where females displayed higher level stress than males. In non-crossnational families, the relationship was reversed $r=-.02(p=.87)$, where females tended to experience less stress than males. Despite the trends, overall, parent gender was not statistically significant in both family types (Table 1; found in the Appendix).

Due to the very weak correlation between parent-level variables (age and gender) and parenting stress, multiple regression analysis consistently showed non-significant results. Table 2 (found in the Appendix) summarizes the influence of predicting variables on parenting stress by family type. In cross-national families, parent age and gender together explained $4.2 \%$ of the variance $\left(R^{2}=.042, F(2,78)=1.73, p=.19\right)$. It was further found that parent age $(\beta=-.11, p=$ $.35)$ and gender $(\beta=.17, p=.14)$ did not significantly predict perceived parenting stress. In contrast, parent age and gender explained a lower percentage of variance of parenting stress $(.6 \%)$ in non-cross-national families $\left(R^{2}=.01, F(2,74)=.22, p=.80\right)$. In addition, parent gender and age were not found to be significant predictors of parenting stress.

Next, stepwise multiple regression analysis was utilized to examine family-level variables (SES, yearly income, child age, number of children) in relation to parenting stress. Table 1 (see Appendix) indicates descriptive statistics for family-level variables related to parenting stress. In cross-national families, parents with more children had a significantly higher degree of stress $(r=$ $.44, p \leq .01)$. Although the rest of family-level variables indicated weak to no relationship with parenting stress, SES and yearly income were negatively correlated with parenting stress. Parents with higher SES scores and yearly income experienced less parenting stress. In non-cross-national families, yearly income was strongly and negatively related to parenting stress $(r=-.44, p \leq .01)$, whereas the number of children only showed a negligible relationship $(r=-.14, p=.39)$. SES and child age showed no and weak relationships with parenting stress. For both types of families, SES and child age were relatively stable factors that both weakly correlated to parenting stress.

In the cross-national group, variables SES, yearly income, child age, and number of children together explained $20.4 \%$ variance $\left(R^{2}=.20, F(4,38)=2.44, p=.06\right)$ (see Table 3 , found in Appendix). Number of children explained 14.7\% variance of parenting stress $\left(R^{2}=.15, F(1\right.$, $38)=7.02, p<.05)$. It was a strong predictor of parenting stress $(\beta=.401, p<.05)$. More children 
Yajuan Xiang et al.

in the cross-national families predicted a higher parenting stress level. All family-level variables explained $25.2 \%$ variance in non-cross-national families $\left(R^{2}=.25, F(4,34)=2.87, p<.05\right)$. Yearly income was found to be a significant predictor of parenting stress $(\beta=-.41, p<.05)$, which explained $15.4 \%$ variance $\left(R^{2}=.15, F(1,37)=7.09, p<.05\right)$. Higher incomes predicted lower stress levels in non-cross-national families.

\section{Cross-National Family-Specific Variables and Parenting Stress}

Past research has identified stressors that are specifically associated with cross-national families including: place of residence/status, language proficiency, years living in US, societal attitude, cultural complexity, and social support. Those stressors were all parent-level variables. Due to the nested and smaller sample in this study, the effect of family-level demographic variables was excluded in this analysis. As previous results indicate, parent age and gender, as parent-level demographic variables, were found non-significant predictors of parenting stress. The effects of those variables were not considered in this analysis.

Table 4 presents descriptive statistics of cross-national family-specific variables. Among the 82 parents from 43 cross-national families, there were 41 native-born and 41 foreign-born spouses. For native spouses, the average parenting stress and social support score was 16.90 (SD $=7.0)$ and $2.9(S D=.74)$. Generally, native spouses scored 3.88 on societal attitude and 2.514 on cultural influence. In contrast, foreign-born spouses reported higher parenting stress (19.73) and lower social support (2.79) than native spouses. In addition, foreign-born spouses were less likely to report societal attitude (3.6) and cultural influence (2.366). Pearson's correlation test (Table 5) showed English proficiency $(r=-.26, p \leq .05)$, cultural influence $(r=.29, p \leq .05)$, and social support $(r=-.25, p \leq .05)$ were weakly related to parenting stress. Parents who had lower English proficiency, higher cultural influence, and less social support tended to have higher parenting stress. Moreover, the relationship between variable status, societal attitude, and years living in the US and parenting stress was negligible. But the relationships were negative with parents who were foreign-born spouses and reported higher societal attitude and lived in the US longer, displaying a tendency to have more parenting stress.

Table 4: Descriptive Statistics of Cross-National Family-Specific Variables Related to Parenting Stress by Status

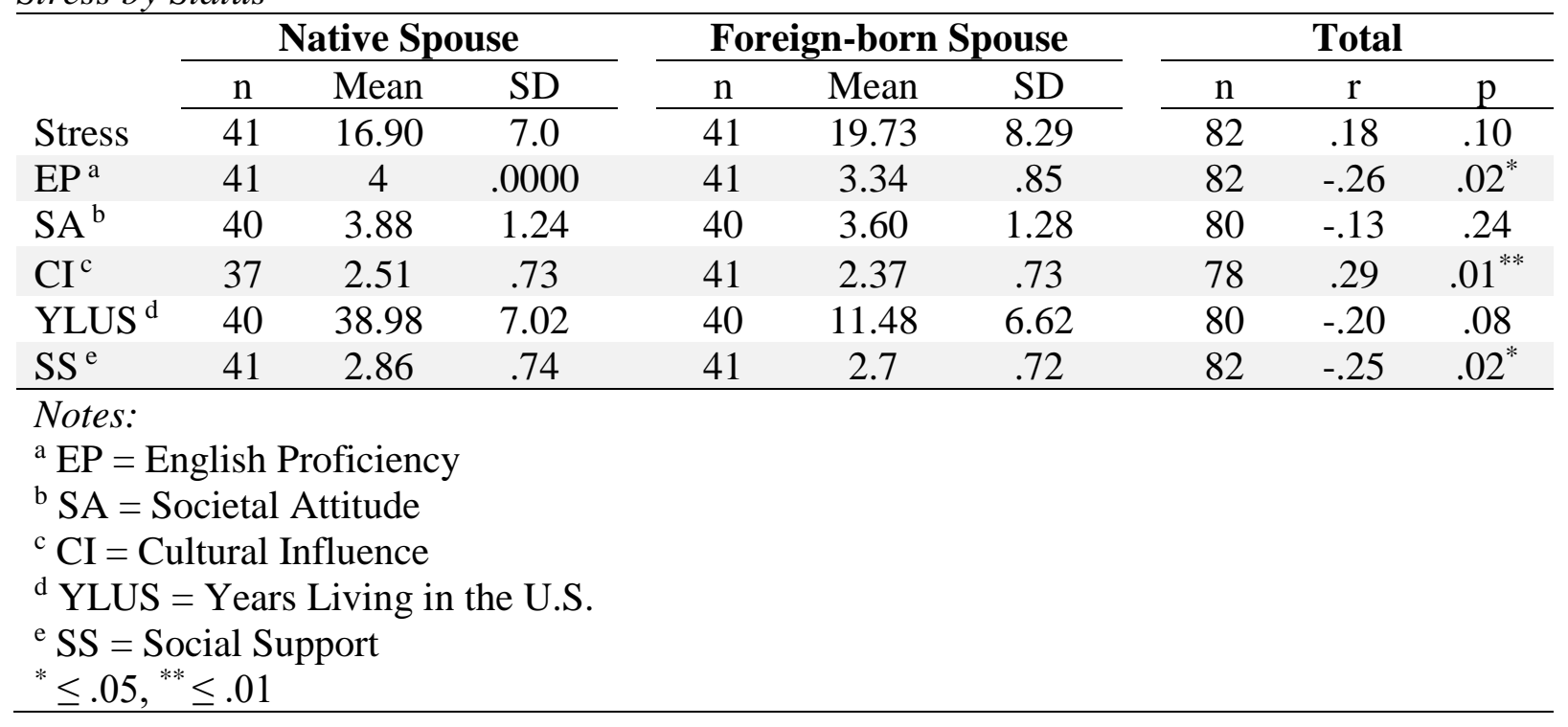


All six cross-national family-specific variables were entered as a cluster in multiple regression analysis. Together, $18.6 \%$ variance $\left(R^{2}=.19, F(6,74)=2.58, p<.05\right)$ of parenting stress was explained. Then six variables were entered in separate steps as indicated in Table 5. The results show, of the total $18.6 \%$ variance, cultural influence itself explains $11.5 \%$ variance of parenting stress. Years living in the US and social support indicate negligible influence on parenting stress. Therefore, the two variables were removed from the stepwise analysis. Status, English proficiency, societal attitude, and cultural influence were then entered separately to further examine the relationship with parenting stress (Table 5). Cultural influence was a stable predictor of parenting stress in cross-national families. It explains $11.2 \%$ variance of parenting stress. Status, English proficiency, and societal attitude account for .02\%variance of parenting stress. Generally, these factors did not influence parenting stress.

Table 5: Summary of Stepwise Multiple Regression Analysis for Cross-national Family-Specific Variables Predicting Parenting Stress

\begin{tabular}{lcccc}
\hline Variable & $\mathbf{B}$ & $\mathbf{B}$ & $\mathbf{\Delta \mathbf { R } ^ { \mathbf { 2 } }}$ & $\mathbf{p}$ \\
\hline Step 1 & .92 & .06 & .02 & .62 \\
Step 2 & -2.68 & -.24 & .02 & .06 \\
Step 3 & -1.00 & -.17 & .02 & .13 \\
Step 4 & 3.58 & .34 & .11 & $.00^{\text {d }}$ \\
\hline Notes: & & & \\
${ }^{\text {a }}$ Step 1: Status & & & \\
${ }^{\mathrm{b}}$ Step 2: Status, English Proficiency & & \\
${ }^{\mathrm{c}}$ Step 3: Status, English Proficiency, Societal Attitude & \\
${ }^{\mathrm{d}}$ Step 4: Status, English Proficiency, Societal Attitude, Cultural Influence \\
${ }^{* *} p<.01$
\end{tabular}

\section{Discussion}

The purpose of this study was to examine whether parents in cross-national families experience different levels of parenting stress compared to their counterparts in non-crossnational families and to better understand possible contributors to parenting stress among crossnational families in the US. Results suggest cross-national couples experience parenting stress similar to those in non-cross-national families. In addition, the number of children and cultural influences are significantly related to parenting stress in cross-national families. More children in a family and more distinct cultural difference within couples contribute to a higher levels of parenting stress among cross-national families.

A major conclusion of past qualitative research on cross-couple populations has been on the unique stressors confronted by couples (Adams, 2004; Bratawidjaja, 2007; Cottrell, 1990; Crippen \& Brew, 2007; Imamura, 1990; Seto \& Cavallaro, 2007; Tosakul, 2010). This study applied statistical analysis and suggest that cross-national couples experience only slightly higher parenting stress than their counterparts in non-cross-national families.

Parent age, gender, SES, yearly income, and child age were not significant predictors of parenting stress. The number of children, however, did contribute to differences in stress levels, which was consistent with previous studies regarding parenting stress (Deater-Deckard, 2004; Skreden et al., 2012). Having more children in the household predicts a higher levels of 
Yajuan Xiang et al.

parenting stress for cross-national couples. Because of the nested data condition and relatively small sample size, the number of children, a family-level variable, was not considered when examining effects of parent-level variables.

Cultural influence was found to be another strong predictor of parenting stress. This result echoes past research where cultural differences not only complicates marital relationships, but raises emotional distress, and eventually might increase parental stress (Baltas \& Steptoe, 2000; Bustamante et al., 2011; Bystydzienski, 2011; Cohen, 1982; Seto \& Cavallaro, 2007). However, the following two aspects are worth noting when interpreting such a result. On the one hand, couples hold a wide range of perceptions regarding cultural influence on parenting. Crippen and Brew (2013) capture a range of perceptions on cultural factors for parenting concerns from minimizing, acknowledging, differentiating and transcending to emphasizing cultural differences in parenting. Thus, it is likely that some effects of cultural influence on parenting stress were not captured in the current study. On the other hand, marriage is a personal affair. Although parenthood may complicate marital relationships (Nyström \& Öhrling, 2004) as it breaks the dyadic balance within couples, parents all tend to restructure their roles and gain a new balance to adapt to the situation (Sevón, 2012). Each spouse within the marriage has a different tolerance for stress and coping strategies (Crippen \& Brew, 2013). The ecology mutually developed by couples is family specific. Thus, the same challenge may not hold across families. There is not enough data to draw the conclusion that experiencing more cultural difference will necessarily lead to more parenting stress. Further, cross-national couples did not report an extreme amount of parenting stress.

Foreign-born spouses reported higher parenting stress than their native-born spouses. However, this might be because female foreign-born spouses made up the majority of the sample and generated a stronger voice. It was not found that being female and of foreign status would predict more parenting stress. This disadvantaged situation for foreign-born spouses was relative within couples. When foreign-born spouses experience difficulties in childrearing in the host culture, the native-born spouses may invest greater efforts to make up the situation in order to maintain a healthy childrearing process. For example, with foreign-born spouses' limited English proficiency, the native-born spouses might take over responsibility for children's education.

A number of limitations should be noted. First, this study proposed to examine potential factors relating to parenting stress among cross-national families. Due to the nested data condition, the sample size did not allow examination of the effect of parent- and family-level variables together. Thus, the effect of the number of children (family-level variable) on parenting stress was not considered when testing cultural influence, a parent-level variable. Second, cultural influence was identified as a significant contributor to parenting stress among crossnational couples. However, the measure of cultural influence on parenting provides limited reliability. Third, participants were recruited through snowball sampling. Hartas (2010) stated that snowball sampling is superior when targeted respondents may not be easily accessible by other means. The sampling method contributed to the relatively homogeneous sample in this study. Participants possessed high SES and English proficiency. For the racial and gender construct, the majority of cross-national couples were of Asian-Western, and female foreignborn and male native-born combinations. The condition of the data does not permit for fine distinctions to be made about the effect of countries of origin and gender. Given the great variation among cross-national families, it could be that the results of this study did not generate a well-rounded image of such families. It would be beneficial for future studies to use a more reliable instrument to measure cultural influence on parenting. A better defined cohort group and 
a larger sample size would allow researchers to examine cross-national couples from different ethnic and cultural groups as an individual unit. Although cross-national marriages are considered the union of individuals from two countries of origin, they are not a homogenous group, as each of them brings along their unique cultural orientation. Thus, the combination of the couple's countries of origin has a great variation. Furthermore, a larger sample would also allow researchers to gain a clearer understanding about the effect of different level of variables on parenting stress.

In conclusion, the current study distinguishes cross-national families from the general intermarriage study and sheds light upon quantitative analysis on their unique stressors in relation to parenting stress in the U.S. No significant differences in parenting stress were found between family types. Number of children and cultural influence, however, were strong predictors. As an exploration study, we suggest future research to take the following four aspects into account: first, demographic variables should be tested repeatedly, especially SES and number of children; second, the different gender and status combinations should be balanced to hear more voices from foreign-born male spouses; third, foreign-born spouses' years living in the US and English proficiency need to be continually investigated; fourth, the moderating and/or mediating effect of social support on parenting stress needs to be examined. It is our hope that this study can be used for researchers and practitioners in education and consulting fields. We encourage more research on this increasing population in not only the couple realm, but more importantly on parent-child and childrearing experiences. Understanding the context of such family will help prepare education and services to children and their families. 
Yajuan Xiang et al.

\section{References}

Adams, J. (2004). "This is not where I belong!" The emotional, ongoing, and collective aspects of couples' decision making about where to live. Journal of Comparative Family Studies, 459-484.

Ali, M. A. (2008). Loss of parenting self-efficacy among immigrant parents. Contemporary Issues in Early Childhood, 9(2), 148-160. doi:10.2304/ciec.2008.9.2.148

Baker, C. K., Perilla, J. L., \& Norris, F. H. (2001). Parenting stress and parenting competence among Latino men who batter. Journal of Interpersonal Violence, 16(11), 1139-1157.

Baltas, Z., \& Steptoe, A. (2000). Migration, culture conflict and psychological well-being among Turkish-British married couples. Ethnicity \& Health, 5(2), 173-180. doi:10.1080/713667445

Baugh, R., \& Witsman, K. (2017). US lawful permanent residents: 2015. Washington, DC: US Department of Homeland Security, Office of Immigration Statistics, 4.

Berry, J. O., \& Jones, W. H. (1995). The parental stress scale: Initial psychometric evidence. Journal of Social and Personal Relationships, 12(3), 463-472. doi:10.1177/0265407595123009

Bhugun, D. (2017). Parenting advice for intercultural couples: a systemic perspective. Journal of Family Therapy, 39(3), 454-477.

Bikel, \& Mandarano, L. (2012). When intercultural relations fail-What happens to the children. Retrieved from https://globenewswire.com/news-

release/2012/06/12/479231/259017/en/When-Intercultural-Relations-Fail-What-Happens-tothe-Children.html

Bratawidjaja, A. (2007). The experience of being parents of mixed-heritage children: Phenomenological analysis. (Doctor of Philosophy Dissertation), Kansas State University, Manhattan.

Breger, R., \& Hill, R. (1998). Cross-cultural marriage: Identity and choice: Bloomsbury Academic.

Bronfenbrenner, U. (1994). Ecological models of human development. Readings on the development of children, 2(1), 37-43.

Bronfenbrenner, U., \& Morris, P. A. (2007). The bioecological model of human development Handbook of Child Psychology: John Wiley \& Sons, Inc.

Bustamante, R. M., Nelson, J. A., Richard C. Henriksen, J., \& Monakes, S. (2011). Intercultural couples: coping with culture-related stressors. The Family Journal, 19(2), 154-164. doi:10.1177/1066480711399723

Bystydzienski, J. M. (2011). Intercultural couples: Crossing boundaries, negotiating difference: NYU Press.

Caballero, C., Puthussery, S., \& Edwards, R. (2008). Parenting "mixed" children: Negotiating difference and belonging in mixed race, ethnicity and faith families. Retrieved from https://www.jrf.org.uk/report/parenting-mixed-children-difference-and-belonging-mixedrace-and-faith-families

Cohen, N. (1982). Same or different? A problem of identity in cross-cultural marriages. Journal of Family Therapy, 4(2), 177-199.

Coll, C. G., \& Pachter, L. M. (2002). Ethnic and minority parenting. Handbook of parenting: Social conditions and applied parenting, 4, 1-20.

Cools, C. A. (2006). Relational communication in intercultural couples. Language and Intercultural Communication, 6(3-4), 262-274.

ISSN 2325-6389 
Cottrell, A. B. (1990). Cross-national marriages: A review of the literature. Journal of Comparative Family Studies, 21(2), 151-169.

Crippen, C., \& Brew, L. (2007). Intercultural parenting and the transcultural family: A literature review. The Family Journal, 15(2), 107-115. doi:10.1177/1066480706297783

Crippen, C., \& Brew, L. (2013). Strategies of cultural adaption in intercultural parenting. The Family Journal, 21(3), 263-271. doi:10.1177/1066480713476664

Deater-Deckard, K. (2004). Parenting stress. Yale University Press.

Djurdjevic, M., \& Roca Girona, J. (2016). Mixed couples and critical cosmopolitanism: experiences of cross-border love. Journal of Intercultural Studies, 37(4), 390-405.

Dunst, C., Jenkins, V., \& Trivette, C. (1984). Family support scale: Reliability and validity. Journal of Individual, Family, and Community Wellness, 1(4), 45-52.

Egan, N. (1995). Crossing cultures: The story of a Chinese man and an American woman. Swaying: Essays on intercultural love, 181-198.

Hartas, D. (2010). Educational research and inquiry: Qualitative and quantitative approaches. Bloomsbury Academic.

Imamura, E. A. (1990). Strangers in a strange land: Coping with marginality in international marriage. Journal of Comparative Family Studies, 21(2), 171-191.

Jambunathan, S., Burts, D. C., \& Pierce, S. (2000). Comparisons of parenting attitudes among five ethnic groups in the United States. Journal of Comparative Family Studies, 31(4), 395406.

Kalmijn, M. (1998). Intermarriage and homogamy: causes, patterns, trends. Annual Review of Sociology, 24(1), 395-421. doi:10.1146/annurev.soc.24.1.395

Kim, B., \& McGoldrick, M. (1998). Marriages of Asian women and American military men. Revisioning family therapy, 309-319.

Kuramoto, M., Koide, T., Yoshida, T., \& Ogawa, E. (2017). Raising multicultural children in Japan: A mixed methods examination of parent-child-society dynamics. Journal of Intercultural Communication Research, 1-25.

Lee, S. M., \& Fernandez, M. (1998). Trends in Asian American racial/ethnic intermarriage: A comparison of 1980 and 1990 census data. Sociological Perspectives, 41(2), 323-342. doi: $10.2307 / 1389480$

Mcfadden, J., \& Moore, J. L. (2001). Intercultural marriage and intimacy: Beyond the continental divide. International Journal for the Advancement of Counselling, 23(4), 261268. doi:10.1023/A:1014420107362

Molina, B., Estrada, D., \& Burnett, J. A. (2004). Cultural communities: Challenges and opportunities in the creation of "Happily Ever After" stories of intercultural couplehood. The Family Journal, 12(2), 139-147. doi:10.1177/1066480703261962

Nyström, K., \& Öhrling, K. (2004). Parenthood experiences during the child's first year: literature review. Journal of Advanced Nursing, 46(3), 319-330. doi:10.1111/j.13652648.2004.02991.x

Romano, D. (2008). Intercultural marriage: Promises and pitfalls: Nicholas Brealey Publishing.

Rosenblatt, P. C. (2009). A systems theory analysis of intercultural couple relationships. Intercultural couples: Exploring diversity in intimate relationships, 320.

Seto, A., \& Cavallaro, M. (2007). Cross-national couples in the mainland United States. The Family Journal, 15(3), 258-264. doi:10.1177/1066480707301315 
Yajuan Xiang et al.

Sevón, E. (2012). "My life has changed, but his life hasn't": Making sense of the gendering of parenthood during the transition to motherhood. Feminism \& Psychology, 22(1), 60-80. doi: $10.1177 / 0959353511415076$

Sinha, R. (1998). The cultural adjustment of Asian lone mothers living in London. London, UK: Ashgate.

Skreden, M., Skari, H., Malt, U. F., Pripp, A. H., Björk, M. D., Faugli, A., \& Emblem, R. (2012). Parenting stress and emotional wellbeing in mothers and fathers of preschool children. Scandinavian Journal of Social Medicine, 40(7), 596-604. doi:10.1177/1403494812460347

Tosakul, R. (2010). Cross-border marriages: Experiences of village women from Northeastern Thailand with Western men. Cross-Border Marriages: Gender and Mobility in Transnational Asia, 179-199.

Turney, K., \& Kao, G. (2009). Barriers to school involvement: Are immigrant parents disadvantaged? The Journal of Educational Research, 102(4), 257-271. doi:10.3200/JOER.102.4.257-271

Wieling, E. (2003). Latino/a and white marriages. Journal of Couple \& Relationship Therapy, 2(2-3), 41-55. doi:10.1300/J398v02n02_04

Yaman, A., Mesman, J., van IJzendoorn, M. H., \& Bakermans-Kranenburg, M. J. (2010).

Perceived family stress, parenting efficacy, and child externalizing behaviors in secondgeneration immigrant mothers. Social Psychiatry and Psychiatric Epidemiology, 45(4), 505-512. doi:10.1007/s00127-009-0097-2

Yang, W.-S., \& Lu, M. C.-W. (2010). Asian cross-border marriage migration: Demographic patterns and social issues (Vol. 2): Amsterdam University Press.

\section{Notes}

${ }^{1}$ China, Malaysia, South Korea, Vietnam, Japan, Philippine, Indonesia, and Iran.

${ }^{2}$ Germany, Czech Republic, Italy, Poland, Sweden, Serbia, and Hungary.

${ }^{3}$ Colombia and Brazil.

${ }^{4}$ Cebuano, Tagalog, Czech, German, Hungarian, Swedish, Portuguese, Indonesia, Farsi, Hokkien, Korean, Vietnamese, Italian, Polish, and Serbian.

${ }^{5}$ Colleges/universities, early childhood facilities, public schools, churches, and parent clubs.

${ }^{6}$ Student associations, community churches, language institutes, and online minority discussion forum. 


\section{APPENDIX}

Table 1: Descriptive Statistics of Variables of Interest Related to Parenting Stress and Social Support by Family Type

\begin{tabular}{|c|c|c|c|c|c|c|c|c|c|c|c|c|c|c|}
\hline & \multicolumn{9}{|c|}{ Cross-National Family } & \multicolumn{5}{|c|}{ Non-Cross-National Family } \\
\hline & $n$ & Mean & $S D$ & $r_{\text {stress }}$ & $p_{\text {stress }}$ & $r_{\text {support }}$ & $\begin{array}{c}p_{\text {supp }} \\
\text { ort }\end{array}$ & $n$ & Mean & $S D$ & $r_{\text {stress }}$ & $p_{\text {stress }}$ & $r_{\text {support }}$ & $p_{\text {support }}$ \\
\hline \multicolumn{15}{|l|}{ PLVa } \\
\hline Parent Age & 81 & 39.09 & 6.42 & -.12 & .28 & -.13 & .24 & 77 & 38.09 & 6.16 & .08 & .51 & .07 & .56 \\
\hline Parent Gender & 82 & $\begin{array}{c}-- \\
17.02 \%\end{array}$ & $\begin{array}{l}-- \\
7.55 \%\end{array}$ & .17 & .12 & -.13 & .26 & 77 & $\begin{array}{c}-- \\
16.76 \%\end{array}$ & $\begin{array}{c}-- \\
7.39 \%\end{array}$ & -.02 & .87 & .03 & .78 \\
\hline Male & 42 & $\begin{array}{l}2.92^{\mathrm{e}} \\
19.68^{\mathrm{c}}\end{array}$ & $\begin{array}{c}.7^{\mathrm{e}} \\
7.81^{\mathrm{c}} \%\end{array}$ & -- & -- & -- & -- & 37 & $\begin{array}{c}2.85^{\mathrm{f}} \\
16.5^{\mathrm{d}} /\end{array}$ & $\begin{array}{l}.57^{\mathrm{f}} \\
6.77^{\mathrm{d}} /\end{array}$ & -- & -- & -- & -- \\
\hline Female & 40 & $2.73^{\mathrm{e}}$ & $.74^{\mathrm{e}}$ & -- & -- & -- & -- & 40 & $2.89^{f}$ & $.61^{\mathrm{f}}$ & -- & -- & -- & -- \\
\hline \multicolumn{15}{|l|}{ FLV $V^{b}$} \\
\hline SES & 43 & 47.27 & 12.25 & -.13 & .41 & -.24 & .12 & 40 & 47.85 & 10.78 & -.12 & .45 & $.40^{*}$ & .01 \\
\hline Yearly Income & 43 & $51,953.59$ & $31,894.05$ & -.03 & .83 & -.27 & .08 & 39 & $55,705.71$ & $23,943.59$ & $-.44^{* *}$ & .01 & .10 & .55 \\
\hline $\begin{array}{l}\text { Child Age } \\
\text { No. of }\end{array}$ & 43 & 4.28 & 3.35 & .2 & .20 & $-.43 * *$ & .00 & 40 & 4.93 & 3.44 & .23 & .16 & .19 & .24 \\
\hline Children & 43 & 1.79 & .71 & $.44^{* *}$ & .00 & -.19 & .21 & 40 & 2.15 & 1.21 & .14 & .39 & -.06 & .72 \\
\hline $\begin{array}{l}\text { Note: } \\
\text { a Parent-level va } \\
{ }^{\mathrm{b}} \text { Family-level va } \\
{ }^{\mathrm{c}} \text { Mean and SD } \\
\mathrm{d} \text { Mean and SD } \\
\text { families. } \\
{ }^{\mathrm{e}} \text { Mean and SD } \\
{ }^{\mathrm{f}} \text { Mean and SD } \\
{ }^{*} p \leq .05,2 \text {-tailec } \\
{ }^{*} p \leq .01,2 \text {-taile }\end{array}$ & $\begin{array}{l}\text { f par } \\
\text { f par } \\
\text { f soc } \\
\text { f soc }\end{array}$ & $\begin{array}{l}\text { s. } \\
\text { nting stres } \\
\text { nting stres } \\
\text { al support } \\
\text { al support }\end{array}$ & $\begin{array}{l}\text { for differ } \\
\text { for differ } \\
\text { or differe } \\
\text { or differe }\end{array}$ & $\begin{array}{l}\text { it gen } \\
\text { gende } \\
\text { gende }\end{array}$ & $\begin{array}{l}\text { er in c } \\
\text { ler in } n \\
r \text { in cro } \\
\text { in cro }\end{array}$ & $\begin{array}{l}\text { oss-natio } \\
\text { n-cross- } \\
\text { s-nation } \\
\text { s-nation }\end{array}$ & $\begin{array}{l}\text { nal fa } \\
\text { hation } \\
\text { a fam } \\
\text { l fam }\end{array}$ & & & & & & & \\
\hline
\end{tabular}


Table 2: Summary of Multiple Regression Analysis for Parent-level Variables Predicting Parenting Stress by Family type

\section{Cross-National Family}

\begin{tabular}{|c|c|c|c|c|c|c|c|c|c|c|c|c|c|c|c|c|}
\hline \multirow[b]{2}{*}{ Variable } & \multicolumn{4}{|c|}{ Parenting Stress } & \multicolumn{4}{|c|}{ Social Support } & \multicolumn{4}{|c|}{ Parenting Stress } & \multicolumn{4}{|c|}{ Social Support } \\
\hline & $B$ & $\beta$ & $R^{2}$ & $p$ & $B$ & $\beta$ & $R^{2}$ & $p$ & $B$ & $\beta$ & $R^{2}$ & $p$ & $B$ & $\beta$ & $R^{2}$ & $p$ \\
\hline Step 1 & -- & -- & .04 & -- & -- & -- & .044 & -- & -- & -- & .01 & -- & -- & -- & .01 & -- \\
\hline Parent Age & -.12 & -.11 & -- & .35 & -.02 & -.15 & -- & .19 & .09 & .08 & -- & .52 & .01 & .08 & -- & .51 \\
\hline Parent Gender & 2.58 & .17 & -- & .14 & -.23 & -.16 & -- & .15 & -.02 & -.00 & -- & .99 & .06 & .05 & -- & .68 \\
\hline
\end{tabular}

Table 3: Summary of Stepwise Multiple Regression Analysis for Family-level Variables Predicting Parenting Stress by Family Type

\section{Cross-National Family}

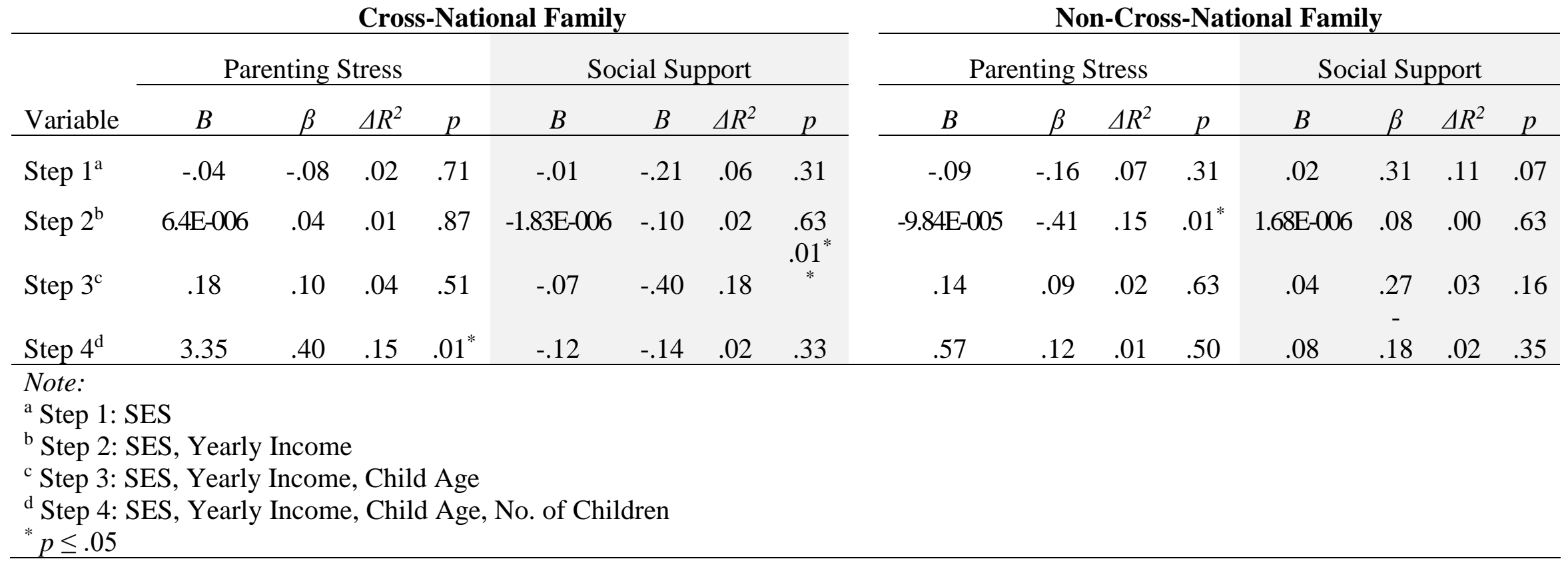

\section{Non-Cross-National Family}

